\title{
Índice de morte neonatal precoce: Uma análise do perfil materno
}

\author{
Early neonatal death index: an analysis in the maternal profile
}

Índice de muerte neonatal precoz: un análisis en el perfil materno

\begin{abstract}
Ana Cristina Farah Abdon da Silva ${ }^{1 *}$, Thayná Martins dos Santos Gonçalves ${ }^{1 *}$, Maria Dolores da Silva ${ }^{1}$, Eduarda Larissa Soares Silva ${ }^{1}$, Maria Isabelle Paixão de Albuquerque ${ }^{1}$, Marcela Carneiro Ferreira Machado de Oliveira ${ }^{1}$, Carolina de Azevedo Nascimento ${ }^{1}$, Maria Inês Bezerra de Melo ${ }^{1}$, Eliana Valentim da Silva ${ }^{1}$, Sandra Cavalcanti Machado do Rêgo Barros ${ }^{1}$; Karla da Silva Ramos ${ }^{1}$, Carina Ribeiro de Oliveira ${ }^{1}$, Cláudia Roberta Selfes de Mendonça ${ }^{1}$, Joanna Francyne Silva de Barros $^{1}$, Nayara Rosane Vasconcelos da Silva ${ }^{1}$.
\end{abstract}

\section{RESUMO}

Objetivo: Identificar os principais fatores de risco que contribuem para a mortalidade precoce a partir do perfil materno. Métodos: Estudo do tipo revisão integrativa. Resultados/Revisão Bibliográficas: Dente os estudos foram selecionados os fatores relacionados ao recém-nascido, que associaram estatisticamente ao óbito neonatal, tais como, o índice de vitalidade neonatal menor que sete, peso ao nascer, inferior a $1.500 \mathrm{e}$ a má formação congênita. No entanto, a patologia neonatal mais importante que contribui para o óbito neonatal foi a sepse $(69,35 \%)$, seguido de choque séptico $(38,7 \%)$. Em relação as características obstétricas, a idade gestacional menor que 30 semanas foi indicativo de prematuridade extrema (56,5\%). E 61,3\% das genitoras encontravam-se em uma faixa etária prevalente de 18 a 28 anos de idade. No Brasil para os coeficientes de mortalidade neonatal precoce, tardio e pós-neonatal foram de 7,3; 6,2 e 2,3/1.000 nascidos vivos, respectivamente. Conclusão: Faz-se necessário reavaliar as intervenções e medidas de saúde adequadas voltadas ao binômio mãe-bebê, para minimizar o impacto desse indicador de saúde.

Palavras-chave: Recém-nascidos prematuros, Gravidez de alto risco, Atenção primária e mortalidade neonatal.

\begin{abstract}
Objective: To identify the main risk factors that contribute to early neonatal mortality from the maternal profile. Methods: Integrative review type study. Results. Among the studies, the factors related to the newborn were statistically associated with neonatal death, such as the neonatal vitality index below seven, the birth weight below $1500 \mathrm{~g}$, and the congenital malformation. However, the most important neonatal pathology that contributed to neonatal death was sepsis $(69.35 \%)$ followed by septic shock (38.7\%). Regarding obstetric characteristics, gestational age less than 30 weeks was indicative of extreme prematurity (56.5\%). And $61.3 \%$ of the mothers were in a prevalent age range of 18 to 28 years of age. In Brazil, the coefficients of early, late and neonatal mortality were 7.3; 6.2 and 2.3/ 1000 live births, respectively. Conclusion: It is necessary to reassess the interventions and adequate health measures aimed at the mother-baby binomial, in order to minimize the impact of this health indicator.
\end{abstract}

Key words: Infant Premature, Pregnancy, High-Risk, Primary Health Care and Infant Mortality.

${ }^{1}$ Faculdade Pernambucana de Saúde (FPS), Cidade: Recife. Estado: PE. * E-mail: ana.cristina@fps.edu.br

SUBMETIDO EM: 3/2019

ACEITO EM: 4/2019

PUBLICADO EM: 7/2019 


\section{RESUMEN}

Objetivo: Identificar los principales factores de riesgo que contribuyen a la mortalidad neonatal precoz, a partir del perfil materno. Métodos: Estudio del tipo revisión integrativa. Resultados Entre los estudios se seleccionaron los factores relacionados con el recién nacido, que se asociaron estadísticamente al óbito neonatal fueron, el índice de la vitalidad menor que siete, el peso al nacer inferior a $1500 \mathrm{~g}$ y la malformación congénita. Sin embargo, la patología neonatal más importante que contribuyó al óbito neonatal fue la sepsis $(69,35 \%)$, seguido de shock séptico (38,7\%). En relación a las características obstétricas, la edad gestacional menor de 30 semanas, fue indicativo de prematuridad extrema (56,5\%). Y el $61,3 \%$ de los progenitores se encontraban en un rango de edad prevalente de 18 a 28 años de edad. En Brasil para los coeficientes de mortalidad neonatal precoz, tardío y post neonatal fueron de 7,3; 6,2 y 2,3 / 1.000 nacidos vivos, respectivamente. Conclusión: Se hace necesario reevaluar las intervenciones y medidas de salud adecuadas, dirigidas al binomio madre-bebé, para minimizar el impacto traído por el indicador de mortalidad neonatal precoz, que aún persiste como relevante problema de salud pública.

Palabras clave: Recién nacidos prematuros, Embarazo de alto riesgo, Atención primaria y mortalidad neonatal.

\section{INTRODUÇÃO}

Os óbitos neonatais são definidos, segundo o Ministério da Saúde por três componentes, o primeiro referese a taxa de mortalidade neonatal precoce, representados pela razão de todos os óbitos ocorridos em recémnascidos na primeira semana de vida do neonato (de 0 ao 6을 dia completo), por mil nascidos vivos, na população residente em um determinado espaço geográfico, por ano considerado; o segundo componente se refere a taxa de mortalidade neonatal tardia, que são as mortes de recém-nascidos que ocorrem entre $07^{\circ} \mathrm{e}$ $27^{\circ}$ dia de vida completo. $\mathrm{E}$ o terceiro se refere à taxa de mortalidade pós-neonatal, que são as mortes ocorridas após 27ํㅡㄹ dia a 364 dias completos (BRASIL, 2017; ROUQUAYROL MZ, 2018).

Embora esse indicador de saúde apresente um perfil nacional de caráter decrescente, ele ainda persiste como um problema de saúde pública no Brasil, em virtude das desigualdades urbanas e das diferenças interregionais ocasionadas pelo indicador de carência social ainda bem presente em diversas regiões do país (MINISTÉRIO DA SAÚDE, 2017).

No Brasil em 2017, ocorreram 36.223 casos de óbitos infantis. Essa distribuição se torna bastante desigual quando comparada às regiões sudeste (13.028) e nordeste (11.490), em relação às do sul (4.034), norte (4.825) e centro-oeste (2.846). Pernambuco registrou 1.352 óbitos e em Recife 429.

Embora estudos tenham sinalizados para uma redução desse indicador de saúde a nível nacional, o ritmo ainda continua abaixo do esperado, os dados são preocupantes quando comparados a dos países desenvolvidos que possuem uma taxa de três a seis vezes menor (UNICEF, 2018), a exemplo a itália, que em 2017 apresentou um dos índices mais baixos de mortalidade infantil do mundo: 3 mortes para cada 1.000 nascidos vivos (SIMEONI S, et al., 2019), em contrapartida, o Brasil, apresentou uma taxa de mortalidade neonatal quatro vezes maior (WHO, 2017; UNICEF, 2018).

Para a United Nations Children'sFund (2017), a prematuridade é um fator de risco bastante relevante para a mortalidade do neonato.De acordo com o último relatório divulgado pelo UNICEF (2018), estatísticas apontam que 60 milhões de crianças morrerão entre 2017 e 2030, antes de completarem cinco anos de vida, sendo metade delas durante o período neonatal. A maioria das mortes de recém-nascidos ocorrerá na Ásia Meridional (39\%), África ao sul do Saara (38\%) e Índia (24\%).

Dados das Organizações das Nações Unidas (ONU), apontam para as disparidades significativas entre países ricos e pobres, a citar, entre os recém-nascidos na África Subsaariana, a cada 1.000 crianças que nascem, 27,77 vão a óbito antes de completarem 7 dias de vida. 
No Brasil a cada 1.000 nascimentos em média 12,39 crianças morrem, enquanto nos países desenvolvidos

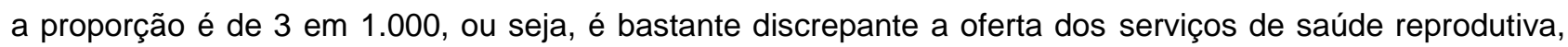
sexual, materna e infantil entre esses países, sendo inconcebível que em 2017, a gravidez e o nascimento da criança ainda sejam condições que ameaçam a vida das mulheres e que 7.000 recém-nascidos morram diariamente por causas evitáveis (WORLD, 2017).

Como a maior parte dos óbitos neonatais ocorrem em regiões de baixa renda, as características que podem estar sendo avaliadas como fator determinante para a perpetuação desse quadro, podem ser tanto as neonatais, tais como, prematuridade, extremo baixo peso ao nascer $(<1000$ gramas), muito baixo peso $(\geq$ 1000 e < 1500 gramas) e baixo peso ( $\geq 1500$ e < 2500 gramas), malformação congênita, asfixia intraparto, infecções perinatais diversas, entre outras (LOURENÇO N, et al., 2017), mas também, podem ser por características maternas e obstétricas, associadas às condições socioeconômicas e de acesso aos serviços de saúde (ZANINI RR, 2011; GAÍVA MA, 2014).

Estudos têm demonstrado dificuldades no itinerário das mulheres com baixa escolaridade, mães sem companheiro, multíparas, gestantes adolescentes (10 a 19 anos), faixas etárias extremas (SANTOS GHN, et al., 2009) ao acesso a atenção básica de saúde, ressaltando a manutenção das iniquidades sociais, étnicos e raciais, especialmente na região norte do país, a exemplo as populações indígenas, que mesmo com os destacados avanços na cobertura da atenção ao pré-natal observados nos últimos anos a rede de serviços de saúde ainda se torna desigual, por se distribuir de forma heterogênea, refletindo na baixa adesão das mulheres ao pré-natal (VIELLAS EF, et al., 2014; TOMASI E, et al., 2017).

Cerca de 70\% das causas de morte neonatal permanecem associadas a fatores reduzíveis (MALTA DC, et al., 2010; NASCIMENTO et al., 2014), por isso, faz-se necessário ampliar a atenção ao pré-natal para que possa garantir a acessibilidade adequada as gestantes e atendimento de qualidade (SÁVIO JM, SANTOS CM, SOUZA RL, TOMAS CD, et al., 2016), voltado a prevenção de riscos na gravidez, detecção e rastreamento precoce das morbidades, redução de complicações no parto, pós-parto e nascimento (KASSAR SB, et al., 2013).

Reduzir a mortalidade neonatal no Brasil tem sido foco constante de intensas reivindicações da população ao longo da história. O sistema de saúde por muitos anos manteve a assistência à criança interligada à saúde materna pela Política de Atenção à Saúde Materno-Infantil (1970).

Em 1984, foi criado o Programa de Assistência Integral à Saúde da Criança (PAISC), considerado um passo importante para o enfrentamento das condições de saúde da população infantil. Logo depois (1991) surgiu o programa de agentes comunitários e o de assistência à saúde perinatal (COSTA R, et al., 2012).

Final da década de 90 foi implantado o programa de referência hospitalar para atendimento as gestantes de alto risco, rumo ao programa nacional de humanização, onde foi também instituída uma norma de atenção humanizada ao recém-nascido de baixo peso: o Método Mãe-Canguru (SILVA AC, et al., 2009; COSTA R, et al., 2012).

Em 2006, através da portaria ํㅡ 399 foi criado o pacto para a saúde, subdividido em pacto em defesa do SUS, de gestão e pacto para a vida, este último, apresenta como uma das prioridades pactuadas entre os gestores: a redução da mortalidade materno-infantil (BRASIL MS, 2006).

Outros programas de saúde foram criados em 2011 e que merecem destaques: Rede Cegonha e o Programa Nacional de Melhoria do Acesso e da Qualidade da Atenção Básica (PMAQ-AB), onde o primeiro programa envolve uma rede de cuidados com vistas a garantir segurança e qualidade assistencial à mulher em todo ciclo reprodutivo, gravídico, puerperal, bem como garantir à criança, a segurança e o cuidado integral ao nascimento, crescimento e desenvolvimento.

Já o segundo, tem como finalidade: ampliar o acesso a atenção primária de saúde, porém, mesmo com todos esses avanços e em virtude de intensas mudanças sociais, econômicas, demográficas, de transição epidemiológica e das situações de vulnerabilidades sociais, o indicador de mortalidade infantil ainda se mantinha em níveis inaceitáveis (MINISTÉRIO DA SAÚDE BR, 2011). 
Frente aos desafios tão abstrusos, visando à qualificação das estratégias voltada a saúde da criança na agenda de saúde pública brasileira, se criou em 2015 a Política Nacional de Atenção Integral à Saúde da Criança, onde um dos eixos estratégicos é a vigilância e prevenção do óbito infantil, fetal e materno (BRASIL MS, 2018). Logo, o objetivo desse estudo foi identificar as principais causas de óbito neonatal precoce, a partir da análise do perfil materno.

\section{METODOLOGIA}

Estudo do tipo revisão integrativa, que foi iniciado a partir da elaboração do objetivo da pesquisa, seguido da formulação de uma hipótese e dos critérios de inclusão e exclusão que ajudaram na seleção dos artigos em bases de dados eletrônicas.

Foi realizado pelos pesquisadores do projeto no período de agosto de 2018 a março de 2019, um levantamento bibliográfico por meio de consulta direta à internet, no endereço eletrônico na plataforma da Biblioteca Virtual de Saúde (BVS), nas seguintes bases de dados: literatura latino-americana e do ensino em ciências da saúde (LILACS), consultadas pela Biblioteca Virtual em Saúde (BVS), e índice cumulativo de literatura de saúde em enfermagem e afins (CINAHL), sistema de análise e recuperação de literatura médica online (MEDLINE), via PUBMED e WEB OF SCIENCE, via Coleção Principal (THOMSON REUTERS SCIENTIFIC), acessadas pelo Portal CAPES.

O software ENDNOTE foi utilizado para gerenciar as referências e importar os artigos das bases de dados, organizando os artigos em grupos denominados: "selecionados para leitura na íntegra" e "excluídos após leitura de título, resumo e artigo completo. Para a pesquisa foram utilizados descritores (MESH/DECS) e termos livres (MESH/TERMS), cruzados através do operador booleano "AND" e "OR" dispostos na Tabela 1.

Tabela 1 - Estratégias de busca dos artigos para consulta em bases de dados eletrônicas.

\begin{tabular}{|c|c|c|}
\hline Cruzamento em Ir & Cruzamento em Espanhol & Cruzamento em Português \\
\hline $\begin{array}{l}\text { Infant, Premature (DECS) AND } \\
\text { Maternal-child nursing (MESH } \\
\text { TERMS), OR Pregnancy, High- } \\
\text { Risk (MESH TERMS). }\end{array}$ & $\begin{array}{l}\text { acido prematuro (DECS) } \\
\text { ermería Materno-infantil } \\
\text { ERMS), OR Embarazo, } \\
\text { go (MESH TERMS). }\end{array}$ & $\begin{array}{l}\text { Recém-nascidor prematuro } \\
\text { (DECS) AND Enfermagem } \\
\text { Materno-infantil } \quad \text { (MESH } \\
\text { TERMS), OR Gravidez, Alto } \\
\text { Risco (MESH TERMS). }\end{array}$ \\
\hline $\begin{array}{l}\text { Pregnancy, High-Risk (DECS) } \\
\text { AND Primary Health Care (MESH } \\
\text { TERMS) OR Infant, Premature } \\
\text { (MESH TERMS). }\end{array}$ & 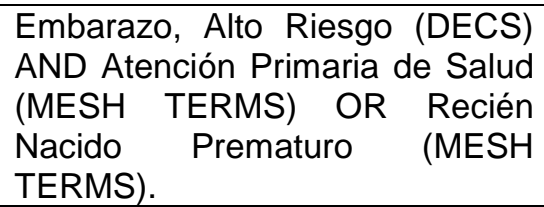 & $\begin{array}{l}\text { Gravidez, Alto Risco (DECS) } \\
\text { AND Cuidados Primários de } \\
\text { Saúde (MESH TERMS) OR } \\
\text { Infantil, Prematuro (MESH } \\
\text { TERMS). }\end{array}$ \\
\hline $\begin{array}{l}\text { Pregnancy, High-Risk } \text { (DECS) } \\
\text { AND Infant Mortality (MESH } \\
\text { TERMS), OR Primary Health Care } \\
\text { (MESH TERMS). }\end{array}$ & $\begin{array}{l}\text { Embarazo, Alto Riesgo (DECS) } \\
\text { AND Mortalidad infantil (MESH } \\
\text { TERMS), OR Atención Primaria a } \\
\text { la salud (MESH TERMS). }\end{array}$ & $\begin{array}{l}\text { Gravidez, Alto Risco (DECS) } \\
\text { AND Mortalidade Infantil } \\
\text { (MESH TERMS), OR } \\
\text { Atenção Primária à Saúde } \\
\text { (MESH TERMS). }\end{array}$ \\
\hline
\end{tabular}

Fonte: Tabela construída pelos autores do projeto, utilizando como fonte as bases de dados LILACS, MEDLINE, BIREME, SCIELO e PUBMED.

${ }^{*}$ Descritores em ciências de saúde; ${ }^{* \star}$ Medical Subject Headings.

Para realização desse trabalho foi incluído artigos originais, disponíveis na íntegra, sobre a temática que contextualize as principais causas de morte neonatal prematura com o perfil materno, sem restrição de idiomas, país de publicação. Foi utilizado um recorte temporal de 2016 a 2019, disponíveis nas bases de dados eletrônicas.

Para os critérios de exclusão, não serão considerados os artigos que fugiram do tema proposto pelos autores, os que se encontravam repetidos nas bases de dados eletrônicas, os manuais técnicos e as revisões de literatura, exceto revisão sistemática em virtude da alta qualidade em evidência científica. 
Para selecionar os artigos, foi necessário que os autores respeitassem os critérios de inclusão e exclusão, caso gerasse alguma dúvida quanto ao objeto de estudo, fez-se necessário que os pesquisadores realizassem a releitura dos títulos, no caso dos títulos comportassem informações insuficientes, se precisou realizar uma releitura dos resumos e descritores, se ainda assim houvesse alguma incerteza sobre sua inclusão, se realizou a leitura completa dos artigos e mesmo diante de todas esssas etapas percorridas e mesmo assim permanecesse a dúvida, se optou pela escolha de dois revisores para segunda e última análise.

Nessa última análise, os dois revisores de forma independente refez todo percursso metodológico acima descrito e de maneira criteriosa, com rigor técnico e conhecimento dos critérios previamente estabecidos, após todas essas etapas rigorosamente percorridas, em caso de dúvida sobre a inclusão dos artigos para composição da amostra final, se utilizou a técnica de consenso.

\section{RESULTADOS}

Inicialmente, foram identificados 1.255 artigos e destes 99 foram selecionados para o estudo, provenientes de periódicos internacionais (31\%) e nacionais (69\%), disponíveis nos bancos de dados eletrônicos. Também foram excluídos os artigos duplicados, ou seja, que se encontrravam repetidos nas bases de dados eletrônicas, como LILACS (7); BDENF (15); WEB OF SCIENCE (5); CINAHL (6); MEDLINE via PUBMED (14); totalizando 47 artigos, além daqueles eliminados por critérios de exclusão e critérios de elegibilidade, obtendose, assim, uma amostra final de cinco estudos nacionais $(n=5)$ referente ao tema do projeto de acordo com a figura 1.

Figura 1 - Fluxograma com estratégias de seleção de artigos, após busca em bases de dados eletrônicos, $2006-2018$.

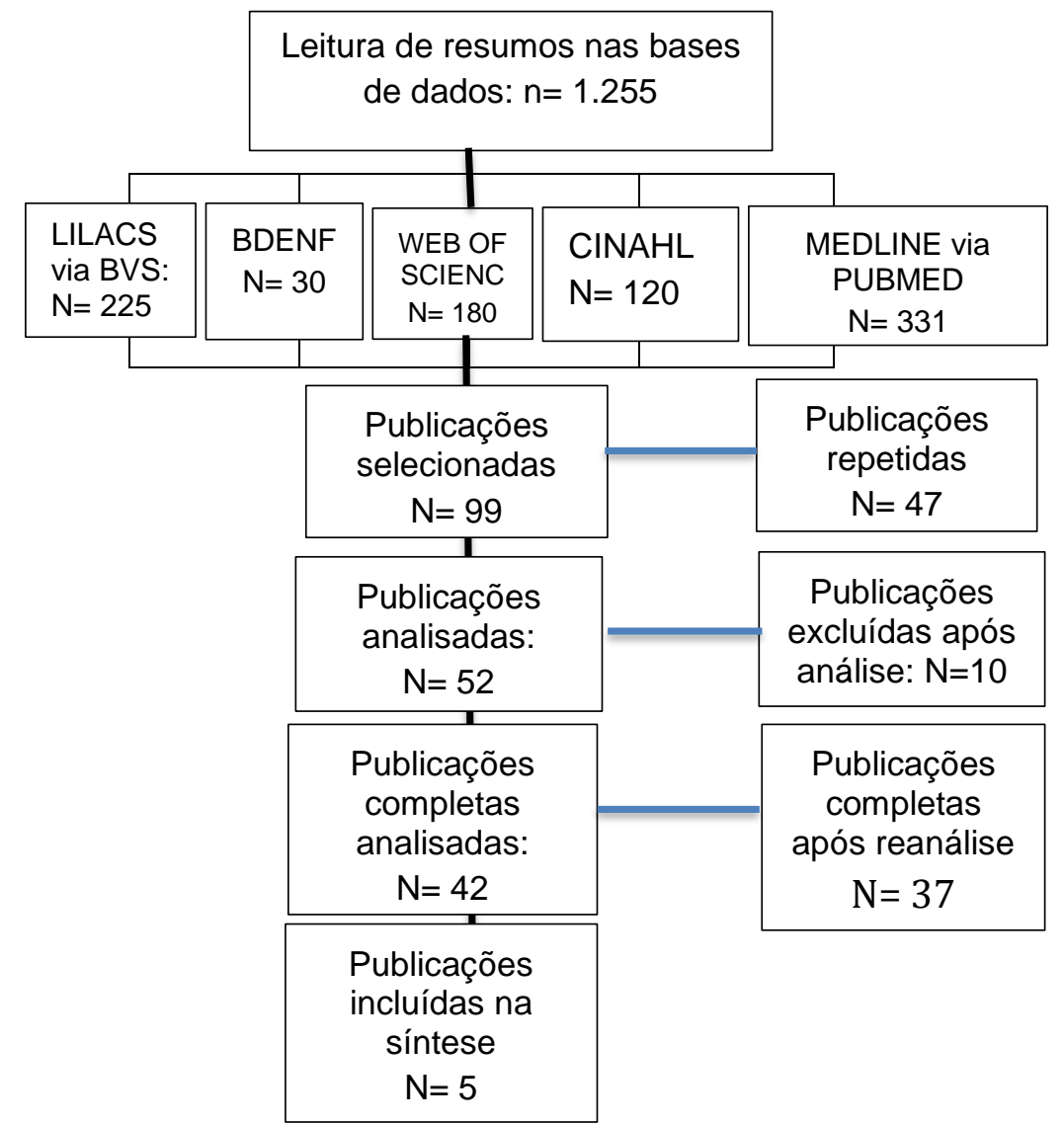

Fonte: Fluxograma construído pelos autores (as) do projeto utilizando como fonte as bases de dados da LILACS, CINAHL, MEDLINE via PUBMED e WEB OF SCIENCE 
As publicações foram analisadas, os dados interpretados de forma organizada e sintetizada por meio da construção de uma tabela com descrição dos seguintes aspectos: Autores/ano, desenho/objetivo metodológico, resultados e conclusão, dispostos na tabela 2. De acordo com a classificação, as pesquisas enquadram-se como artigos originais $(n=5)$, sendo todos de abordagem quantitativa, porém apresentando diferentes desenhos metodológicos.

Tabela 2 - Análise de artigos sobre mortalidade neonatal precoce e fatores de risco materno-infantil, 2016 - 2019.

\begin{tabular}{|c|c|c|}
\hline Autores/ano & Metodologia & Objetivo \\
\hline $\begin{array}{ll}\text { Rodrigues } & \text { NCP; } \\
\text { Monteiro } & \text { DLM: }\end{array}$ & $\begin{array}{l}\text { Estudo usou } \\
\text { técnicas de análise }\end{array}$ & $\begin{array}{l}\text { Avaliar as tendências } \\
\text { de mortalidade }\end{array}$ \\
\hline $\begin{array}{l}\text { Almeida AS; Barros } \\
\text { MBL, et al, (2016). }\end{array}$ & $\begin{array}{l}\text { espacial com } \\
\text { registros de óbito } \\
\text { do Sistema de } \\
\text { Informações sobre } \\
\text { Mortalidade. }\end{array}$ & $\begin{array}{l}\text { materna e neonatal } \\
\text { espacial e temporal } \\
\text { no Brasil entre } 1997 \text { e } \\
2012 \text {. }\end{array}$ \\
\hline
\end{tabular}

Kale PL; Mello MHP;
Silva KS; Fonseca
SC (2017).
Silva KS;
SC (2017)

Estudo prospectivo
de coorte de
nascimentos com
base hospitalar.

Avaliar os fatores associados a casos de ${ }^{* * *}$ near miss materna, neonatal e óbitos neonatais em seis maternidades públicas de São Paulo e Rio de Janeiro, em 2011. Analisar os fatores de risco para Mortalidade Neonatal Precoce, segundo os critérios de estratificação de risco.

Resultados
$27,08 \%$ de 1990 a
$2012 . \quad$ Em
contrapartida a **TMM
aumentou $11 \%$ de
2009 a 2012 . Já os
óbitos neonatais
precoces caíram 33\%.
Foram observadas
taxas altas de ${ }^{* \star}$ near
miss para sífilis
materna, falta de
atendimento pré-natal,
cor materna preta,
falta de pré-natal.
Conclusões

A ${ }^{\star}$ TMN do Brasil
melhorou nos últimos anos, mas a materna não. As políticas públicas e os esforços entre setores poderão contribuir para as melhorias dos indicadores.

São necessárias melhoras no acesso ao atendimento

qualificado para gestantes e recémnascidos para reduzir os riscos à vida dos neonatos.

\begin{tabular}{ll}
\hline Migoto MT, Oliveira & Estudo \\
RP, Silva AMR, & epidemiológico do \\
Freire MHS (2018). & tipos caso- \\
& controle.
\end{tabular}

\section{Fatores de risco Estudo inovador de maternos: fatores de risco ao escolaridade e perdas óbito neonatal precoce} fetais; neonatais: na perspectiva da sexo, baixo peso ao Linha Guia, nascer, prematuridade,

${ }^{* * \star *}$ APGAR menor que 7 no quinto minuto e assistenciais: até seis consultas.

Canuto IMB; Alves
FAP; Oliveira CM; et
al, (2019)

Estudo do tipo
Ecológico,
realizado
Recife

Analisar a distribuição espacial intraurbana da mortalidade perinatal, a capacidade de sua prevenção e a sua relação com os fatores socioeconómicos na cidade de Recife, no período entre 2013 a 2015.

Teixeira JAM; Araújo
WRM; Maranha descritivo.
AGK, et al, 2019.

Calcular taxas de
mortalidade no
primeiro dia de vida
entre 2010 e 2015 em
oito Unidades da
Federação brasileira
com melhor qualidade
de informação.

Verificaram-se clusters mortalidade perinatal em bairros das regiões Sul e Sudoeste. Com exceção da região Norte e Nordeste por identificar bairros com maior carência social (pobreza). instrumento

tecnológico de gestão para saúde materna e infantil, na busca de sua qualificação e maior sensibilidade

A estratificação do espaço urbano, de acordo com o indicador de carência social, apresentou estreita relação com a mortalidade perinatal por causas evitáveis. Mostra diferenças intraurbanas quanto ao óbito perinatal.

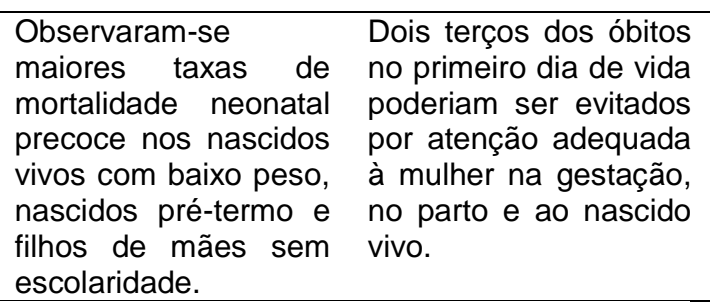
Fonte: Tabela elaborada pelos (as) autores (as) do projeto, utilizando fontes das bases de dados LILACS, CINAHL, MEDLINE, via PUBMED e WEB OF SCIENCE.

* Taxa de Mortalidade Infantil Neonatal; ** Taxa de Mortalidade Materna; ${ }^{* * *}$ Estado de disfunção grave ou falha total do organismo-morte; ${ }^{* * * *}$ índice de vitalidade neonatal. 


\section{DISCUSSÃO}

O Brasil apresenta níveis alarmantes e estatisticamente inaceitáveis de mortalidade infantil e relacionados a problemas perinatais, nos quais algumas sub-regiões apesar de taxas decrescentes nos últimos dez anos a nível nacional, ainda têm muito a ser feito (CANUTO IMB, 2019).

Outro estudo, anterior a esse, também afirma que, globalmente, a redução do coeficiente de mortalidade infantil (IMC), por si, é baixa, de forma desigual, segundo o grau de desenvolvimento de cada região, o que se reflete nas desigualdades sociais existentes (MEDEIROS ZM, 2016), onde a região norte (reduziu de 27,8 para 13,3), em relação à região Sul (reduziu 17,8 para 13,8), segundo dados do DATASUS (2016).

Percebem-se, ainda, as marcantes desigualdades do CMI por regiões. Dos 5.565 municípios brasileiros, 250 apresentam CMI maior que 40/1.000 nascidos vivos (NV), sendo que 40\% são localizados na Região Nordeste. Por outro lado, nas regiões Sul e Sudeste, mais de 80\% dos municípios têm CMI inferior a 20/1.000 nascidos vivos (ALMEIDA CA; RODRIGUES EM, 2014 e MEDEIROS ZM, 2016).

De acordo com a Organização das Nações Unidas (2011), dos oitos objetivos de desenvolvimento do milênio, cuja meta era de reduzir até 2015 a mortalidade infantil em dois terços em relação aos níveis de 1990, além de reduzir a fome e a miséria, foi cumprida pelo Brasil antes do previsto (KROPIWIEC MV; FRANCO SC; AMARAL AR, 2017), porém quando se faz um retrato por estados, como São Paulo, Piauí e Pernambuco, verificou-se que CMI por 1.000 NV foi de 11,6; 16,9 e 18,1, respectivamente (ALMEIDA, WS 2014).

Observa-se, portanto, a forma desigual da redução do indicador de saúde, realidade que foi evidenciada em outros estudos (BARROS FC, 2010; COSTA JP, 2011; RODRIGUES M, 2014).

Esses dados supracitados, também apresentam características semelhantes, quando comparados aos estudos realizados na Bahia e em Minas Gerais no período de 2000 a 2012 (TAVARES LT, 2016; FARIA CS, 2014), por apresentar a mesma situação de declínio em relação à TMI. Mesmo com esse avanço no país, a mortalidade infantil, segundo a UNITED NATIONS CHILDREN'S FUND (2015), mostra uma taxa de 15,7 mortes por mil nascimentos vivos, o que coloca o país no $90^{\circ}$ lugar entre 187 países no ranking da Organização das Nações Unidas (ONU), atrás de Cuba $(5,25)$, Chile $(6,48)$, Argentina $(12,8)$, China $(15,4)$ e México $(16,5)$.

Alguns estudos mostram que indicador de saúde ainda mantém um caráter persistente, sendo um dos motivos, o quadro de iniquidade social, levando aos óbitos neonatais a serem considerados como principal componente da mortalidade no primeiro ano de vida (UNICEF, 2017; CANUTO IMB, et al., 2019), dados esses também enfatizados em outro estudo ao retratar que o país pouco apresentou mudanças nos principais determinantes sociais e na organização dos serviços de saúde, quanto ao acesso a maioria das intervenções em saúde materno-infantil, devido a maior complexidade dos fatores biológicos, socioeconômicos e assistenciais, além de outros fatores determinantes relacionado as características clínicas e epidemiológicas do binômio mãe-filho (LEAL MC, et al., 2018).

Estudos recentes têm mostrado que mães jovens, com idades extremas, negras, solteiras, com história reprodutiva desfavorável, baixo nível de instrução, usuárias de drogas, com diagnóstico clínico de diabetes gestacional e pré-eclâmpsia, obesidade, múltiplas gestações, anomalia fetal e abortos foram fatores associados a gestação de alto risco, as complicações obstétricas-neonatais e a maior ocorrência de prematuridade (AQUINO PT; SOUTO BGA, 2015 e SAMPAIO AF, 2018).

Segundo Fonseca SC et al. (2017), o efeito combinado de apenas dois desses fatores supracitados (extremos de idade e baixa escolaridade) tem aumentado em cerca de $70 \%$ a chance de óbito neonatal, posto que a idade materna também é fator determinante de complicações durante o período gravídico, com a ocorrência de pré-eclâmpsia, doença essa considerada primeira causa de morte materna no Brasil, devido as precárias condições econômicas, culturais e assistenciais (AMORIM FCM, et al., 2017).

No que se refere às condições socioeconômicas desfavoráveis, como baixa escolaridade e baixa renda familiar, têm levado mulheres à gestação de alto risco, o que compromete o desenvolvimento intrauterino, 
podendo levar a óbitos fetais, visto que essas situações estão associadas, em geral, ao estresse, a piores condições nutricionais e a baixa procura ao atendimento de pré-natal (SILVA LS, 2018), este último, muito vivenciado por mães negras por apresentarem dupla situação de vulnerabilidade, ora pela exclusão histórica marcada pelo preconceito, ora pela ausência de ações específicas para a superação da desigualdade (THEOPHILO RL; RATTNER D e PEREIRA EL, 2018.

O planejamento reprodutivo, o diagnóstico precoce de doenças e o tratamento eficaz das complicações que podem surgir durante a gravidez, no parto e no pós-parto, quando acompanhados na atenção primária de saúde, tendem a reduzir os óbitos tanto maternos, como neonatais, além de assegurar o direito fundamental do ser humano que é o direito à vida (FONSECA SC, et al., 2017).

No entanto, estudos têm demonstrado uma associação significativa entre os óbitos neonatais com a precária assistência ao pré-natal e atenção inadequada aos recém-nascidos nas salas de parto das maternidades (CASTRO EC, 2007; BARROS FC, 2010 e NASCIMENTO RM, et al., 2012), mostrando-se em consonância com a pesquisa de FESCINA RH et al. (2011), do qual afirma-se, que, deve-se buscar promover a saúde materna e fetal principalmente na fase em que antecede ao parto, além de rastrear situações de risco e tratar intercorrências mais precocemente possível.

Esse tipo de cuidado melhora os desfechos clínicos na gestação e no puerpério, além de influenciar na redução da mortalidade materno-infantil e perinatal (BRASIL MS, 2018).

Quanto aos óbitos perinatais, Lassi ZS e Bhutta ZA (2015) também retrataram a faixa etária materna entre 20 e 34 anos e escolaridade maior que oito anos como elemento relevante, afora os níveis socioeconômicos mais baixos, de grupos étnicos específicos, baixo grau de educação e de pessoas que vivem em áreas carentes. Os fatores que contribuem para a mortalidade perinatal incluem mau passado obstétrico, curto intervalo intraparto, gravidez múltipla, menor idade gestacional, história de natimorto, hipertensão, diabetes e ausência de cuidados pré-natais (DEMITTO MO; GRAVENA AAF e ANTUNES MB, 2017).

Por outro lado, segundo Costa DK (2016), a assistência pré-natal se faz presente em todas as Estratégias de Saúde da Família, onde é reconhecida como a porta de entrada de principal escolha da gestante no sistema único de saúde, que se estende, desde o atendimento de suas necessidades, ao acompanhamento continuado proporcionado pela atenção básica durante a gravidez, objetivando diminuir o número de nascimento no período pré-termo, ou seja, no período menor que 37 semanas de gestação.

O aumento de nascimentos prematuros tem sido relacionado a diversos fatores, a saber, os novos métodos de fertilização, com o consequente aumento no número de gravidez gemelar; o crescente número de gestações no grupo de mulheres com mais de 35 anos; o aumento das indicações médicas de partos cesáreos, geradas pelo maior emprego da tecnologia no acompanhamento da gestação (BRASIL, 2016).

No Brasil, a partir da implantação da Política Nacional de Humanização ao Parto, Puerpério e Nascimento, criado em 2003, a cobertura e o número médio de consultas da atenção pré-natal apresentam tendência crescente. Entretanto, pouco se sabe sobre a avaliação da qualidade do pré-natal na maioria dos estudos, porém há indícios relevantes de que a baixa qualidade seja um problema mais grave do que simplesmente a realização de um menor número de consultas preconizadas pelo MS (2011).

Sabe-se que a qualidade da assistência realizada durante o pré-natal pode reduzir de $10 \%$ a $20 \%$ a quantidade de óbitos nesse período (NEUMANN NA, 2003), corroborando com outras pesquisas, em que os dados apontam que a adequada atenção pré-natal, tem se apresentado como um dos principais fatores de proteção, não somente contra a mortalidade nessa população em estudo, mas também contra o baixo peso ao nascer, a prematuridade e o retardo do crescimento intrauterino (DARMSTADT GL, 2005; BARROS FC, 2008).

Essa pesquisa apresentou convergência relacionada a outro achado científico, quanto à dificuldade de se reconhecer a causa dos partos prematuros, por conta da baixa adesão ao controle de pré-natal, visto que $86,1 \%$ das mulheres não haviam realizado um número mínimo de consultas igual ou superior a seis (MINISTÉRIO DA SAÚDE BR, 2011), fator este relacionado a outro estudo internacional, em que se relatou 
uma associação entre número insuficiente de consultas pré-natais e aumento de mortes neonatais por até 11,6 vezes maiores (GAÍVA MA, 2013). O controle realizado no pré-natal é imprescindível para detecção precoce de intercorrências e realização de ações de prevenção de doenças. É o momento de reconhecer doenças que podem acometer o feto e a mãe durante a gestação, as quais podem levar à prematuridade extrema e consequentemente morte neonatal (RODRIGUES M, 2011).

Recente estudo considera que tanto o acesso, como a qualidade dos serviços de saúde são impactados pelos fatores socioeconômicos e culturais de uma população e isso reflete não somente em prejuízo à saúde materna, fetal e neonatal, como também, pode resultar em maiores taxas de mortalidade perinatal e neonatal. Essa situação determina maior atenção, em especial nas regiões de maior carência social (MIGOTO MT, 2018).

O coeficiente neonatal é reconhecido como um dos mais sensíveis indicadores de saúde de uma população, pois a morte de crianças menores de um ano é diretamente influenciada por fatores de risco, como condições de pré-natal, gravidez, história materna, doenças maternas, ruptura precoce de membrana, gemelaridade, consanguinidade, síndrome da morte súbita, estado marital, diferenças raciais maternas e infantis, condições socioeconômicas, prematuridade, baixo peso ao nascer, más formações congênitas, mães portadoras do HIV e de outras doenças infectocontagiosas, dentre outras (BRASIL MS, 2009; BORBA GC, 2013; (DEMITTO MO, 2017).

Para tanto, faz-se necessário, identificar também outros fatores relacionados ao parto, tais como, idade gestacional, condições e tipo de parto, intervalo intraparto, hemorragia perinatal; mortalidade perinatais e fatores relacionados ao nascimento, como, índice de $A P G A R$, asfixia, hipóxia, persistência do canal arterial (PCA), termorregulação ineficaz, contribuindo para maior tempo de hospitalização dos recém-nascidos (RN) em unidade de terapia intensiva neonatal, ente outros (SILVEIRA RC, 2013; LANSKY SC, 2014).

Os achados do presente estudo mostraram que as elevadas taxas de morbimortalidade dos recémnascidos pré-termos, ainda persistem como um grande desafio na assistência neonatal. $E$ as vertentes entre as causas da prematuridade e as principais complicações, necessitam de intervenções seguras, bem como, de ações que contribuam para redução das desigualdades na saúde, favorecendo maior sobrevida neonatal.

\section{CONSIDERAÇÕES FINAIS}

Estudos evidenciam a necessidade de rever o acesso aos serviços de saúde em todo território brasileiro, assim como a qualidade da assistência no pré-natal, e também, as condições de assistência ao parto e dos cuidados imediatos após o nascimento. Todo esse panorama tem demonstrado a importância de intervenções na redução da mortalidade infantil, por ainda persistir como um grande desafio na assistência maternoneonatal.

\section{REFERÊNCIAS}

1. ALMEIDA WS, SZWARCWALD CL. Mortalidade infantil nos municípios brasileiros: uma proposta de método de estimação. Rev Bras Saude Mater Infant, 2014; 14(4): 331-342.

2. APARECIDA MG, et al. Mortalidade neonatal em crianças com baixo peso ao nascer. Revista de Enfermagem USP, 2014; 48(5): 778-86.

3. BITTENCOURT RM, GAÍVA MAM. Mortalidade neonatal precoce relacionada a intervenções clínicas. Brasília. Revista Brasileira de Enfermagem, 2014; 67(2): 195-201.

4. BORBA GG, et al. Fatores associados à morbimortalidade neonatal: um estudo de revisão. Saúde (Santa Maria), Rio Grande do Sul, 2014; 40: 09-14.

5. BRASIL. BANCO DE DADOS DO SISTEMA ÚNICO DE SAÚDE - DATASUS. Painel de monitoramento da mortalidade infantil, 2017.

6. BRASIL. MINISTÉRIO DA SAÚDE. A vigilância do óbito no Brasil: trajetória de 2008 a 2015. In: Brasil. Ministério da Saúde. Saúde Brasil 2014: uma análise de situação de saúde e das causas externas. Brasília: Ministério da Saúde, 2015: 45-68.

7. BRASIL. MINISTÉRIO DA SAÚDE. SECRETARIA DE ATENÇÃO À SAÚDE. DEPARTAMENTO DE AÇÕES PROGRAMÁTICAS ESTRATÉGICAS. Política Nacional de Atenção Integral à Saúde da Criança: orientações para implementação/Ministério da Saúde. Secretaria de Atenção à Saúde. Departamento de Ações Programáticas Estratégicas. - Brasília: Ministério da Saúde, $2018: 180$. 
8. BRASIL. Ministério da Saúde; Secretaria de Ciência, Tecnologia e Insumos Estratégicos, Departamento de Ciência e Tecnologia. Síntese de evidências para Políticas de Saúde: mortalidade perinatal [Internet]. Brasília; 2012: 43.

9. CANUTO IMB, et al. Diferenciais intraurbanos da mortalidade perinatal: modelagem para identificação de áreas prioritárias. Recife. Escola Anna Nery 2019; 23(1): 1-8.

10. DARMSTADT GL, et al. Evidence-based, cost-effective interventions: how many newborn babies can we save? Lancet. 2005; 365:977-88.

11. DATASUS. MINISTÉRIO DA SAÚDE. Sistema de Informações sobre Mortalidade neonatal, 2017.

12. DEMITTO MO, et al. Gestação de alto risco e fatores associados ao óbito neonatal. Rev. Da escola de enfermagem da USP, 2017; 51.

13. FARIA CS, et al. Morbidade e mortalidade entre recém-nascido de risco: uma revisão bibliográfica. Enfermería Global. Murcia, 2014; 36 (13): 311-322.

14. FUNDO DAS NAÇÕES UNIDAS PARA A INFÂNCIA (UNICEF). Committing to child survival. A Promise Renewed Progress Report, New York,: Unicef, 2013.

15. GAÍVA MAM, et al. Mortalidade neonatal análise das causas evitáveis. Rio de Janeiro. Revista enfermagem, 2015; 23(2):247-53.

16. GHOJAZADEH M, et al. Contributing death factors in very low-birth-weight infants by path method analysis. Niger Med J. 2014; 55(5): 389-93.

17. ILIODROMITI S, et al. Apgar score and the risk of cause-specific infant mortality: a population-based cohort study. Lancet. 2014: 14(9956):1749-55.

18. KALE PL, et al. Neonatal near miss and mortality: factors associated with life-threatening conditions in newborns at six public maternity hospitals in Southeast Brazil. Cad. Saúde Pública, 2017; 33(4): 1-10.

19. KROPIWIEC MV, et al. Fatores associados à mortalidade infantil em município com índice de desenvolvimento humano elevado. Rev. paul. pediatr. [online]. v.35. 2017; 391-398.

20. LANSKY S, et al. Pesquisa Nascer no Brasil: perfil da mortalidade neonatal avaliação da assistência a gestante e do recém-nascido. cad. Saúde pública. Rio de Janeiro, 2014; 30: S 192-S207.

21. LASSI ZS, HAIDER BA; BHUTTA ZA. Community-based intervention packages for reducing maternal and neonatal morbidity and mortality and improving neonatal outcomes. Cochrane Database Syst Rev, 2016; 10 (11): 1-33.

22. LIMA MCBN, et al. A desigualdade espacial do baixo peso ao nascer no Brasil. Ciênc. Saúde Coletiva. 2013; 18(8):2443-52.

23. LOURENÇO N, et al. Morbidade neonatal dos recém-nascidos pré-termo tardios comparados aos de termo precoce. Sci Med [Internet], 2017; 27(1): 1-13.

24. MALTA DC, et al. Atualização da lista de causas de mortes evitáveis por intervenções do Sistema Único de Saúde do Brasil. Epidemiol Serv Saúde. 2010;19(2):173-6.

25. MEDEIROS ZM, et al. Infant mortality: temporal trend and contribution of death surveillance. Acta Paul. Enferm, $2016 ; 29$.

26. MELO WF, et al. As principais afecções respiratórias no neonato. Revista Brasileira de Educação e saúde. Paraíba, 2015; 5(3):3642.

27. MENDES KDS, et al. Revisão integrativa: método de pesquisa para a incorporação de evidências na saúde e na enfermagem. Texto Contexto Enferm. Florianópolis: 2008; 17(4): 758-64.

28. MIGOTO MT, et al. Mortalidade neonatal precoce e fatores de risco: estudo caso-controle no Paraná. Rev Bras Enferm [Internet]. 2018; 71(5):2527-34.

29. MINISTÉRIO DA SAÚDE (BR); SECRETARIA DE VIGILÂNCIA EM SAÚDE. Saúde Brasil 2013: uma análise da situação de saúde e das doenças transmissíveis relacionadas à pobreza. Brasília (DF): MS; 2014.

30. MOREIRA KFA, et al. Perfil e evitabilidade de óbito neonatal em um município da Amazônia Legal. Cogitare Enfermagem. 2017; 22(2):1-4.

31. OLIVEIRA CM, et al. Vigilância do óbito infantil no Recife, Pernambuco, Brasil: Operacionalização, potencialidades e limites. Epidemiol. Serv. Saude, Brasília, 2017; 26(2):413-419.

32. RODRIGUES M, et al. Spatial analysis of infant mortality and the adequacy of vital information: a proposal for defining priority areas. Ciênc. Saúde Coletiva. 2014; 19(7):2047-54.

33. RODRIGUES NCP, et al. Temporal and spatial evolution of maternal and neonatal mortality rates in Brazil, 1997-2012. J Pediatr (Rio J). 2016; 92(6):567-573.

34. ROLNIK DL, et al. Predição do parto prematuro: avaliação sequencial do colo uterino e do teste para proteína-1 fosforilada ligada ao fator de crescimento insulina-símile. Rev Bras Ginecol Obs. 2013; 35 (9): 394-400.

35. SÁVIO JM, et al. Perfil Clínico de Neonatos internados em uma UTI do Sul Catarinense. Revista Inova Saúde. Santa Catarina, v.5, Secretaria Estadual de Saúde, História do Hospital Barão de Lucena, 2016.

36. SHARMA S, et al. Analytical study of intrauterine fetal death cases and associated maternal conditions. Int J Appl Basic Med Res [Internet]. 2016/2018; 6(1):11-3.

37. TAVARES LT, et al. Mortalidade infantil por causas evitáveis na Bahia, 2000-2012. RECIIS - Rev Eletron Comun Inf Inov Saúde [Internet]. 2016; 10(3):1-10.

38. TEIXEIRA GA, et al. Fatores de risco para a mortalidade neonatal na primeira semana de vida. Revista de Pesquisa cuidado é fundamental online. Rio de Janeiro, 2016; 8(1): 4036-45.

39. UNITED NATIONS INTER-AGENCY GROUP FOR CHILD MORTALITY ESTIMATION (UN IGME), 'Levels \& Trends in Child Mortality: Report 2017, Estimates Developed by the UN Inter-Agency-Group for Child Mortality Estimation', United Nations Children's Fund, New York, 2017.

40. World Health Organization. Estimates Developed by the UN Inter-Agency-Group for Child Mortality Estimation. Report, 2017. 